\title{
Abordaje asterional mínimamente invasivo para descompresión microvascular en neuralgia del trigémino
}

\author{
Ramiro López-Elizalde ${ }^{1,2}$, Esteban Reyes-Velasco', Álvaro Campero³, Pablo Ajler ${ }^{4}$, \\ Karla Cecilia Cornelio-Freer y Marisol Godínez-Rubi i, 5* \\ ${ }^{1}$ Dirección Normativa en Salud, Instituto de Seguridad y Servicios Sociales de los Trabajadores del Estado, Ciudad de México, México; ${ }^{2}$ Servicio \\ de Neurocirugía, OPD Hospital Civil Juan I. Menchaca, Guadalajara, Jalisco, México; ${ }^{3}$ Servicio de Neurocirugía, Hospital Padilla, Tucumán, \\ Argentina; ${ }^{4}$ Departamento de Cirugía Neurológica, Hospital Italiano, Buenos Aires, Argentina; ${ }^{5}$ Departamento de Microbiología y Patología, Centro \\ Universitario de Ciencias de la Salud, Universidad de Guadalajara, Guadalajara, Jalisco, México
}

\section{Resumen}

Objetivo: La neuralgia del trigémino (NT) es un trastorno neuropático susceptible de tratamiento quirúrgico. El objetivo es presentar los hallazgos quirúrgicos y resultados obtenidos en 26 pacientes con NT, tratados mediante un abordaje asterional mínimamente invasivo para descompresión vascular trigeminal. Métodos: Estudio longitudinal descriptivo. Se intervino mediante abordaje asterional a 26 pacientes. Se registró el historial médico, hallazgos quirúrgicos, respuesta al tratamiento y complicaciones. Se les dio seguimiento durante 36 meses. Resultados: Diecinueve casos se asociaron a compresión vascular, cinco casos a aracnoiditis y los dos restantes se relacionaron con esclerosis múltiple y neuralgia postherpética. El dolor se controló significativamente en todos los pacientes durante el postoperatorio inmediato. A 36 meses de seguimiento, en 25 pacientes se alcanzó un control total o aceptable del dolor. A largo plazo 22 pacientes evolucionaron sin complicaciones permanentes. Conclusiones: La cirugía de descompresión microvascular a través de un abordaje asterional mínimamente invasivo para el tratamiento de la NT es una alternativa con resultados similares al abordaje retrosigmoideo clásico, pero que suma las bondades de una técnica quirúrgica que se rige con los principios de la mínima invasión. Se requieren esfuerzos constantes para optimizar las técnicas quirúrgicas en el tratamiento de la NT.

PALABRAS CLAVE: Neuralgia del trigémino. Cirugía de descompresión microvascular. Neurocirugía de mínima invasión. Abordaje asterional.

\section{Abstract}

Objective: Trigeminal neuralgia (TN) is a neuropathic disorder that can be treated surgically. This study aimed to present the surgical findings and the clinical outcomes of 26 patients with TN treated by minimally invasive asterional surgery. Methods: Longitudinal descriptive study. Twenty-six patients with TN underwent minimally invasive asterional surgery. The medical history, surgical findings, therapeutic response, and complications were registered. They were followed for 36 months. Results: Nineteen cases were associated with vascular compression; five were associated with arachnoiditis. The two remaining cases were associated with multiple sclerosis and post-herpetic neuralgia. The pain was substantially reduced in all patients in the immediate postoperative period. At 36 months, in 25 patients, total or acceptable pain control was achieved. In the long term, 22 patients evolved with no permanent complications. Conclusion: The microvascular decompression surgery by an asterional approach is an alternative with similar results to the classic retrosigmoid approach to treat $T N$, but that adds the benefits of the principles of minimally invasive surgery. Constant efforts need to be made to optimize minimally invasive surgical techniques for TN.

KEY WORDS: Trigeminal neuralgia. Microvascular decompression surgery. Minimally invasive neurosurgery. Asterional approach.

Correspondencia:

*Marisol Godínez-Rubí

E-mail: jmarisol.godinezrubi@gmail.com
Fecha de recepción: 06-03-2019

Fecha de aceptación: 19-03-2019

DOI: 10.24875/GMM.19005130
Gac Med Mex. 2019;155(Suppl 1):S70-S78

Disponible en PubMed

www.gacetamedicademexico.com 


\section{Introducción}

La neuralgia del trigémino (NT) es un trastorno neuropático paroxístico que afecta a una (o a todas) las ramas sensoriales del nervio trigémino ${ }^{1,2}$. Diversos estudios revelan una incidencia global de aproximadamente 12.6-28.9 casos por cada 100.000 individuos por año ${ }^{3}$. El mecanismo fisiopatológico de esta entidad es incierto en la mayoría de los casos. Se acepta como la causa más común la compresión por un vaso sanguíneo cercano, más frecuentemente la arteria cerebelosa superior (ACS) $)^{4,5}$.

El tratamiento inicial siempre es médico, pero el paciente puede eventualmente requerir un tratamiento percutáneo, rizotomía o cirugía de descompresión microvascular (DMV) 6 . La DMV del nervio trigémino es un tratamiento efectivo y aceptado para el tratamiento de la NT. Fue realizado por primera vez por Frazier en 1915 mediante un abordaje transtemporal. Posteriormente fue mejorado por Dandy en 1934, quien propuso un abordaje vía subcerebelosa. Gardner Miklos renovó el interés en la cirugía de DMV en $1962^{1,7}$; sin embargo, fue Jannetta quien popularizó la técnica utilizada en la actualidad ${ }^{7}$, .

Para mejorar los resultados postoperatorios, algunos autores han optado por refinar la técnica quirúrgica de DMV-15. En este trabajo presentamos nuestra experiencia en el tratamiento quirúrgico de la NT mediante de un abordaje asterional mínimamente invasivo para descompresión vascular trigeminal.

\section{Material y métodos}

\section{Pacientes}

Se realizó un estudio descriptivo longitudinal que incluyó 26 pacientes con NT que habían tenido síntomas de discapacidad recurrentes y persistentes a pesar del tratamiento médico convencional durante al menos dos años, o aquellos que mostraron intolerancia a la medicación. Todos los casos se sometieron a cirugía de mínima invasión mediante microcraniectomía asterional. La información recabada incluyó edad, sexo, tiempo de evolución, historial médico, características del dolor, hallazgos quirúrgicos, complicaciones y evolución postoperatoria.

\section{Evaluación prequirúrgica}

Previo a la cirugía, todos los pacientes se sometieron a un examen neurológico completo y a una resonancia magnética (RM) craneal en secuencia FIESTA. En la RM se descartaron daños estructurales y se localizaron estructuras anatómicas relevantes como la raíz dorsal del nervio trigémino, la ACS, la vena petrosa, la arteria cerebelosa anteroinferior (ACAI), las cisternas del ángulo pontocerebeloso (APC), el canal auditivo interno y los nervios craneales VII y VIII, así como la arteria timpánica (Fig. 1A). También se identificaron los siguientes puntos de referencia: meato acústico externo, la porción mastoidea del hueso temporal y su neumatización, la cresta supramastoidea, líneas nucales superiores e inferiores y el espacio aracnoideo en la fosa cerebelosa.

\section{Descripción de la técnica quirúrgica}

Se realiza una lista de control del material quirúrgico, así como de la técnica anestésica, el lado que se va a operar (previamente marcado) y de todos los insumos para la cirugía. El paciente se coloca en posición prona (tres cuartos) con la cabeza girada hacia el lado opuesto y el cuello ligeramente flexionado, el hombro ipsilateral se fija hacia abajo y hacia delante, las rodillas y caderas se flexionan y el brazo se coloca $90^{\circ}$ perpendicular al suelo (posición de Park Bench) (Fig. 1B). La cabeza del paciente se fija con cabezal de Mayfield y se gira $45^{\circ}$ contralateralmente hacia el lado afectado por la neuralgia con una flexión ligera (Fig. 1C). La mesa se inclina para elevar el cabezal. Se prepara la zona de interés mediante afeitado y técnica aséptica. Se realiza una incisión paramediana vertical o curvilínea entre las líneas cigomática y mastoides, cruzando el asterión (Fig. 1C). La piel se incide profundamente hasta la fascia muscular, se debe evitar el uso del electrocauterio monopolar. La selección del punto preciso de la incisión cutánea y de la craneotomía se apoya en la información proporcionada por los sistemas de neuronavegación, lo que nos permite obtener una visión exacta de la relación anatómica que guarda el asterión con el seno transverso y el seno sigmoideo. Una vez identificado el punto asterional ${ }^{16,17}$ (Fig. 1D), se realiza una microcraneotomía de aproximadamente $21 \mathrm{~mm}$ (rango de $18-25 \mathrm{~mm}$ ) (Fig. $1 \mathrm{E} \mathrm{y} \mathrm{F}$ ). Se debe evitar acceder a las celdas mastoideas, si esto sucede, deben ser cuidadosamente selladas con cera para hueso.

El margen superolateral de la craneotomía debe coincidir con el punto de unión del borde inferior de los senos transverso y sigmoideo. La duramadre se 

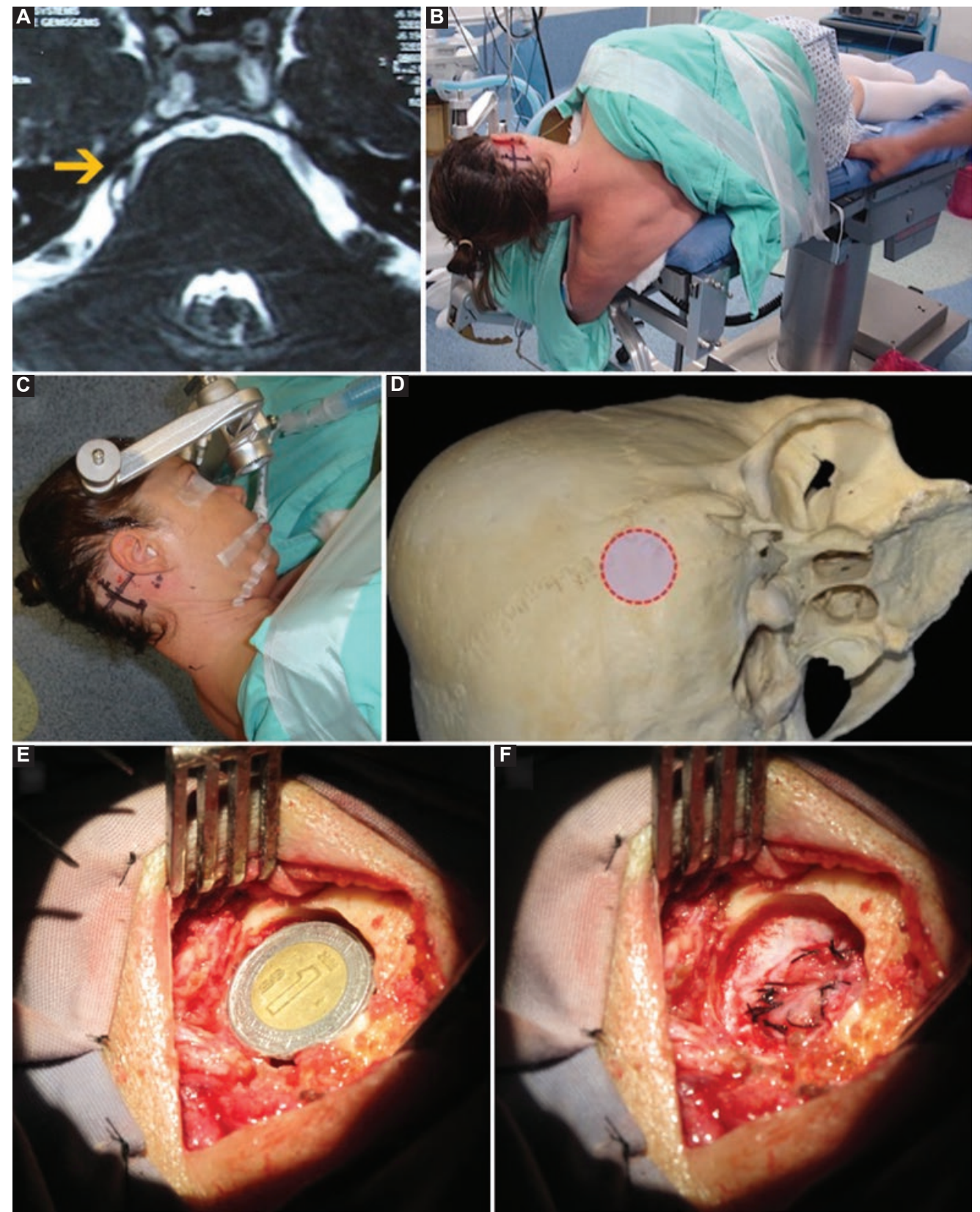

Figura 1. A: secuencia FIESTA ponderada en T2 de RM preoperatoria que muestra un asa vascular de la ACAl ejerciendo compresión sobre el nervio trigémino (flecha amarilla). B: el paciente se coloca en posición de Park Bench, en decúbito lateral con rodillas y caderas en flexión y el brazo contralateral en posición perpendicular al suelo. C: fijación de la cabeza con cabezal de Mayfield con giro de $45^{\circ}$ contralateral al lado afectado; la incisión cutánea es curvilínea y se realiza entre la línea cigomática y la línea mastoidea. D: modelo de la craneotomía (línea punteada) realizada posteroinferior al Asterión; en esta posición, el borde superior de la craneotomía se ubica sobre los senos transverso y sigmoideo. E: se ilustra el tamaño de la ventana ósea $(18-25 \mathrm{~mm})$, con una moneda de $21 \mathrm{~mm}$ utilizada como comparación. F: tamaño de la craneotomía y cierre dural después de concluir el procedimiento.

abre de forma curvilínea, siguiendo los márgenes de la craneotomía, $2 \mathrm{~mm}$ por debajo del seno transverso y $2 \mathrm{~mm}$ por debajo del seno sigmoideo.

Después de la apertura dural, se realiza un drenaje de líquido cefalorraquídeo (LCR) de las cisternas magna y del APC bajo aspiración suave. No se realiza drenaje lumbar de forma sistemática. Si el cerebelo obstruye el campo quirúrgico, se coloca suavemente un separador para contenerlo.

Una vez expuesto el APC, el vector del microscopio quirúrgico se dirige hacia este, con énfasis en el sector superior. Es necesario visualizar el nervio trigémino a lo largo de su trayecto cisternal completo (Fig. 2) para poder disecar la aracnoides que lo 

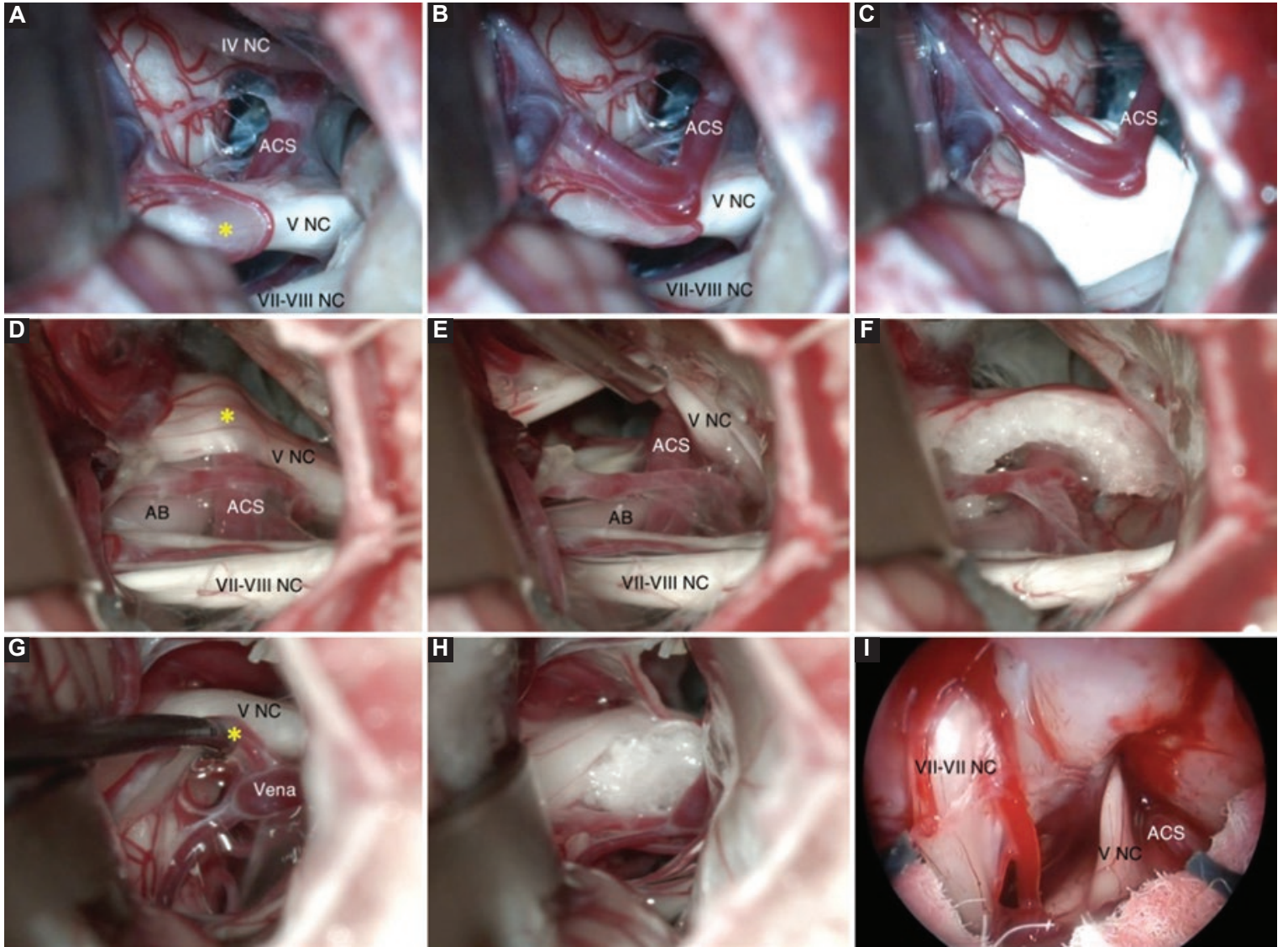

Figura 2. Exposición intradural de estructuras cerebrovasculares dentro de la cisterna cerebelopontina a través de un abordaje asterional mínimamente invasivo en tres pacientes distintos. Paciente 1, figuras A, B y C. A: vista del campo quirúrgico después de la apertura dural y el drenaje de las cisternas. El nervio trigémino se encuentra delante de un asa de la ACS con la que establece un contacto evidente (*) y se adhiere por aracnoides. B: nervio trigémino y ACS una vez que las adherencias han sido liberadas por la remoción del tejido aracnoideo. C: el nervio y la arteria se han separado colocando material aislante entre ellos. Paciente 2, figuras D a F. D: el nervio trigémino pasa por encima de la ACS con contacto entre ellos. E: una vez elevado el nervio, se aprecia la estrecha relación entre ambas estructuras. F: interposición total del material aislante para evitar una mayor compresión del nervio trigémino. Paciente 3, figuras $G$ y $H$. En este caso, dos vasos (arteria y vena) están adyacentes al nervio trigémino (G). H: separación de las estructuras vasculares y nerviosas después de la disección aracnoidea. I: vista endoscópica una vez que el nervio es liberado. Muestra la estrecha relación entre el haz nervioso formado por los nervios craneales VII-VIII y el complejo formado por el nervio trigémino y la ACS. Los asteriscos marcan el punto en el que la estructura vascular hace contacto (anterior 0 posterior) en cada caso con el nervio trigémino.

AB: arteria basilar; ACS: arteria cerebelosa superior; IV NC: cuarto nervio craneal (nervio troclear); V NC: quinto nervio craneal (nervio trigémino); VII NC: séptimo nervio craneal (nervio facial); VIII NC: octavo nervio craneal (nervio vestibulococlear).

cubre en toda su extensión (Fig. 2 A-G). Debe evitarse cualquier manipulación del complejo neurovascular de los nervios craneales VII y VIII para evitar complicaciones.

Una vez que el nervio trigémino es expuesto, cualquier estructura vascular (arterial o venosa) que lo comprima debe ser identificada y alejada del nervio (Fig. 2 B, E y G). Para evitar el contacto entre el nervio y el vaso sanguíneo, se inserta un material aislante entre ellos (Fig. $2 \mathrm{C}, \mathrm{F}$ y H) y, si es necesario, se fija con pegamento de fibrina. Si no se logra visualizar algún contacto vascular, el procedimiento puede ser asistido por un neuroendoscopio (Fig. 2l).
Una vez verificada la hemostasia, se irriga la herida, se cierra la duramadre aproximando los bordes durales, se suturan el pericráneo y el músculo con seda y suturas continuas (Fig. 1F). Cuando está disponible, el colgajo óseo se coloca en su lugar para cerrar la craneotomía; de lo contrario, se puede utilizar una placa de Gelfoam ${ }^{\circledR}$ para cubrir la abertura del cráneo. Los músculos y la piel se suturan. Finalmente la piel se cubre con un apósito estéril.

Cuidados postoperatorios: antibiótico durante 24 horas, así como vigilancia de fugas de LCR, hemorragia o edema del tronco encefálico y del cerebelo. Se prescriben esteroides orales durante una 
Tabla 1. Escala para evaluación de la respuesta terapéutica

\begin{tabular}{|l|l|l|}
\hline Categoría & Estado & Descripción \\
\hline A & Sin dolor & $\begin{array}{l}\text { Sin episodios de neuralgia o crisis de dolor durante el periodo comprendido entre la cirugía } \\
\text { y cada evaluación. }\end{array}$ \\
\hline B & Buen control del dolor & $\begin{array}{l}\text { Uno o más episodios de dolor durante el periodo comprendido entre la cirugía y cada } \\
\text { evaluación, en el mismo dermatomo. El dolor no es incapacitante ni afecta actividades } \\
\text { cotidianas. El dolor responde satisfactoriamente a analgésicos no esteroideos. }\end{array}$ \\
\hline C & Leve control del dolor & $\begin{array}{l}\text { Uno o más episodios de dolor durante el periodo comprendido entre la cirugía y cada evaluación, } \\
\text { en el mismo dermatomo. El dolor no es incapacitante ni afecta actividades cotidianas. El dolor se } \\
\text { alivia con el mismo tratamiento farmacológico que tenía antes de la cirugía. }\end{array}$ \\
\hline D & Falla en el control del dolor & $\begin{array}{l}\text { Dos o más episodios de dolor incapacitantes o que interrumpen actividades cotidianas } \\
\text { durante el periodo comprendido entre la cirugía y cada evaluación. El dolor amerita el } \\
\text { mismo tratamiento que tenía antes de la cirugía. }\end{array}$
\end{tabular}

semana. Los analgésicos junto con los antineuríticos se retiran gradualmente en un periodo de 7 a 15 días.

\section{Seguimiento}

Los pacientes fueron evaluados cada cuatro meses durante el primer año postoperatorio y después de forma anual. La efectividad del control del dolor neuropático se evaluó con base en una escala creada por nuestro equipo de trabajo y aplicada con éxito a todos nuestros pacientes. En esta escala se consideraron cuatro niveles de respuesta, que se designaron por letras (A-D) en orden ascendente (Tabla 1). En cada evaluación postoperatoria se interrogó a los pacientes sobre la presencia de crisis de dolor, su gravedad, la respuesta al tratamiento farmacológico y el impacto en su calidad de vida. La ausencia total de episodios neurálgicos, durante el periodo comprendido entre la cirugía y cada momento de la evaluación, se calificó como categoría «A». Por el contrario, el fracaso total del tratamiento quirúrgico con episodios de dolor de frecuencia o intensidad similar a los anteriores a la cirugía se consideró categoría «D». Las etapas intermedias entre estas categorías se evaluaron como se describe en la tabla 1. También se registraron complicaciones.

\section{Resultados}

\section{Demografía y características de TN}

Veintiséis pacientes fueron incluidos en el estudio (17 mujeres, 9 hombres). La edad media fue de 60.3 años (rango: 34-82 años). Los casos de NT asociados con compresión vascular fueron 19 (73.1\%), dos casos $(7.7 \%)$ se asociaron con aracnoiditis relacionada con neurocisticercosis. Los casos restantes

\section{Tabla 2. Características clínicas y hallazgos quirúrgicos}

\begin{tabular}{|c|c|}
\hline Característica & n (\%) \\
\hline $\begin{array}{l}\text { Periodo bajo tratamiento farmacológico } \\
\text { prequirúrgico } \\
\text { 2-3 años } \\
\text { 3-5 años } \\
\text { >5 años }\end{array}$ & $\begin{array}{l}20(77 \%) \\
3(11.5 \%) \\
3(11.5 \%)\end{array}$ \\
\hline $\begin{array}{l}\text { Lateralidad de la neuralgia } \\
\text { Derecha } \\
\text { Izquierda } \\
\text { Bilateral }\end{array}$ & $\begin{array}{c}17(65.5 \%) \\
8(30.7 \%) \\
1(3.8 \%)\end{array}$ \\
\hline $\begin{array}{l}\text { Dermatomos dolorosos } \\
\text { V1 } \\
\text { V2 } \\
\text { V1 }+ \text { V2 } \\
\text { V2 }+ \text { V3 } \\
V 1+V 2+V 3\end{array}$ & $\begin{array}{c}3(11.5 \%) \\
6(23.1 \%) \\
1(3.8 \%) \\
14(53.9 \%) \\
2(7.7 \%)\end{array}$ \\
\hline $\begin{array}{l}\text { Hallazgos quirúrgicos } \\
\text { Compresión por ACS } \\
\text { Compresión por ACAl } \\
\text { Compresión por complejo venoso } \\
\text { Compresión por la vena petrosa superior } \\
\text { Aracnoiditis } \\
\text { Ninguno }\end{array}$ & $\begin{array}{l}11(42.3 \%) \\
5(19.2 \%) \\
2(7.7 \%) \\
1(3.8 \%) \\
5(19.3 \%) \\
2(7.7 \%)\end{array}$ \\
\hline
\end{tabular}

ACAI: arteria cerebelosa anteroinferior; ACS: arteria cerebelosa superior.

( $n=4,15.3 \%$ ) se asociaron con lesión cerebral traumática, hemorragia subaracnoidea por aneurisma, esclerosis múltiple y neuralgia postherpética (un caso cada uno, respectivamente). La mayoría de los pacientes $(n=20,77 \%)$ recibieron tratamiento previo de tipo farmacológico durante 24-36 meses. El resto $(n=6,23.1 \%)$ tuvo antecedentes de tratamiento médico durante más de 36 meses. La carbamazepina fue el fármaco de uso más frecuente $(n=14$; $53.9 \%)$, seguida de la oxcarbazepina $(n=5,19.2 \%)$. Ninguno de los pacientes tuvo lesiones estructurales identificadas en la RM. El dolor se reportó con más frecuencia en el lado derecho $(n=17,65.5 \%)$. La localización más frecuente del dolor fue una 
Tabla 3. Efectividad del tratamiento quirúrgico a los 36 meses. Hallazgos quirúrgicos y evolución clínica

\begin{tabular}{|c|c|c|c|c|c|c|}
\hline & \multirow[t]{2}{*}{$n(\%)$} & Sexo n (\%) & \multirow[t]{2}{*}{ Edad (años) } & \multirow{2}{*}{$\begin{array}{l}\text { Hallazgos } \\
\text { quirúrgicos }\end{array}$} & \multirow[t]{2}{*}{ Complicaciones temporales } & \multirow{2}{*}{$\begin{array}{l}\text { Complicaciones } \\
\text { permanentes }\end{array}$} \\
\hline & & $\begin{array}{l}\text { Mujer } \\
\text { Hombre }\end{array}$ & & & & \\
\hline A & $18(69.2 \%)$ & $\begin{array}{l}12(46.1 \%) \\
6(23.1 \%)\end{array}$ & $60.4(34-82)$ & $\begin{array}{l}\text { Arteria } 11(42.3 \%) \\
\text { Vena } 3(11.5 \%) \\
\text { Aracnoiditis } 3(11.5 \%) \\
\text { Ninguno } 1(3.8 \%)\end{array}$ & $\begin{array}{l}\text { Cefalea } \\
1(3.8 \%) \\
\text { Fístula LCR } \\
1(3.8 \%) \\
\text { Hipoacusia } \\
2(7.7 \%) \\
\text { HSA } \\
1(3.8 \%) \\
\text { Parálisis facial } 1(3.8 \%) \\
\text { Síndrome cerebeloso } 1(3.8 \%)\end{array}$ & $\begin{array}{l}\text { Hipoacusia } 1(3.8 \%) \\
\text { Tinitus } 1(3.8 \%)\end{array}$ \\
\hline B & $7(27 \%)$ & $\begin{array}{l}5(19.2 \%) \\
2(7.7 \%)\end{array}$ & $60.3(46-76)$ & $\begin{array}{l}\text { Arteria } \\
4(15.4 \%) \\
\text { Vena } 0(0 \%) \\
\text { Aracnoiditis } 2(7.7 \%) \\
\text { Ninguno } 1(3.8 \%)\end{array}$ & $\begin{array}{l}\text { Infección de herida } \\
1(3.8 \%) \\
\text { Isquemia cerebral } \\
1(3.8 \%)\end{array}$ & $\begin{array}{l}\text { Parálisis facial } 1(3.8 \%) \\
\text { S. Millard-Gubler } 1(3.8 \%)\end{array}$ \\
\hline C & $1(3.8 \%)$ & $\begin{array}{c}0(0 \%) \\
1(3.8 \%)\end{array}$ & 60 & $\begin{array}{l}\text { Arteria } \\
1(3.8 \%)\end{array}$ & $\begin{array}{l}\text { Nistagmus } \\
1(3.8 \%)\end{array}$ & - \\
\hline D & $0(0 \%)$ & - & - & - & - & - \\
\hline
\end{tabular}

afectación simultánea de las ramas V2 y V3 ( $\mathrm{n}=14$, $53.9 \%$ ) (Tabla 2).

\section{Hallazgos intraoperatorios}

La arteria que con mayor frecuencia provocó compresión del nervio trigémino fue la ACS $(n=11$, $42.3 \%$ ), seguido de la ACAl y de aracnoiditis ( $n=5$, $19.2 \%$, respectivamente). En tres casos (11.5\%) se documentó únicamente compresión venosa (Tabla 2, Fig. 2). En dos pacientes no hubo compresión vascular ni ningún otro hallazgo que explicara la neuralgia; estos casos tenían antecedentes de esclerosis múltiple e infección por el virus herpes zóster, respectivamente. En todos los casos se diseccionó el nervio y se colocó el material aislante. En 12 pacientes $(46.1 \%)$ el procedimiento quirúrgico fue asistido con endoscopia.

\section{Seguimiento y evaluación de los resultados terapéuticos}

El dolor se redujo significativamente en todos los pacientes durante el periodo postoperatorio inmediato. Con base en la escala propuesta (Tabla 1), se encontró que a los cuatro meses después de la cirugía, y con reevaluaciones posteriores durante al menos 36 meses, se logró la curación absoluta en 18 pacientes $(69.2 \%$, clase A). Siete pacientes $(27 \%)$ tuvieron un control aceptable del dolor $(7.27 \%$, clase B). Solo un caso reportó persistencia de episodios dolorosos que, aunque el dolor disminuyó en frecuencia e intensidad, ameritó el mismo tratamiento farmacológico prequirúrgico (clase $\mathrm{C}$ ) (Tabla 3).

\section{Complicaciones postoperatorias}

El $62 \%$ de los pacientes $(n=16)$ cursaron sin complicaciones tempranas ni tardías. Las complicaciones postoperatorias inmediatas incluyeron dos casos de pérdida de audición (7.7\%), uno de los cuales se resolvió en los primeros 12 meses, y el otro con pérdida permanente. Otro paciente desarrolló una infección de herida quirúrgica, con una complicación de parálisis facial permanente (House-Brackmann III). Un paciente presentó isquemia delimitada del tronco encefálico, que causó síndrome protuberancial con parálisis cruzada (Síndrome de Millard-Gubler). Estos casos demostraron una recuperación significativa con rehabilitación un año después de la cirugía. También hubo un caso de cada uno de los siguientes (todos tratados y con resolución temprana sin complicaciones permanentes): hemorragia subaracnoidea supratentorial, parálisis facial (House-Brackmann 
II), nistagmo, síndrome cerebeloso, cefalea y fístula del LCR. A largo plazo, 22 pacientes evolucionaron sin complicaciones permanentes (84.6\%). No se registró ninguna muerte asociada con el procedimiento (Tabla 3).

\section{Discusión}

Con el advenimiento del microscopio quirúrgico, Janetta pudo implementar con éxito la cirugía de $\mathrm{DMV}^{7,8}$ que hasta la fecha ha sido el método más popular y con mejores resultados para el tratamiento de la $\mathrm{NT}^{6,18,19}$. Desde entonces, se ha buscado perfeccionar la técnica quirúrgica para minimizar el daño por el abordaje y de esta forma prevenir complicaciones. Es por ello que se han descrito gran cantidad de variantes de la cirugía de DMV para el tratamiento de la NT, pero ninguna es perfecta ${ }^{9-15}$. En este manuscrito, presentamos nuestra experiencia en 26 casos sometidos a cirugía de descompresión vascular trigeminal mediante un abordaje asterional mínimamente invasivo.

\section{Técnica quirúrgica}

Los resultados quirúrgicos dependen en gran medida de la colocación del paciente en una posición adecuada (Fig. 1 B y C). La posición lateral permite una visibilidad óptima de la zona quirúrgica en dirección rostro-caudal, facilita la exposición del complejo neurovascular trigeminal sin afectar el retorno venoso y evita el sobreestiramiento del cuello, lo que reduce la frecuencia de dolor postoperatorio $0^{9,12,15,19}$. En cuanto a la incisión cutánea, se debe considerar la localización de los nervios occipital y auricular para evitar su lesión y con ello, el dolor postoperatorio local $^{9}$. Al disecar los tejidos blandos, coincidimos con Tomasello, et al. (2016), quienes enfatizan evitar el uso del electrocauterio monopolar, ya que daña significativamente los vasos sanguíneos, los nervios y los músculos? Este daño produce atrofia, fibrosis y retracción posterior, lo que contribuye a la morbilidad permanente del paciente. En nuestra serie, ningún paciente reportó complicaciones significativas debido al daño cutáneo o muscular.

El abordaje asterional permite practicar craneotomías pequeñas, de forma que hemos sido capaces de trabajar de forma óptima con un diámetro medio de la ventana ósea de $21 \mathrm{~mm}$ (rango: 18-25 mm). Este campo permite una visualización adecuada, lo que evita la necesidad de una amplia extirpación de tejido. Es fundamental evitar acceder a las células mastoides, ya que su apertura aumenta el riesgo de fístula del LCR y de procesos infecciosos graves ${ }^{9,15,19}$. Una vez que el cerebelo está expuesto, se debe evitar el uso de retractores, los cuales pueden promover contusiones, hematomas o edemas cerebeloso. Sin embargo, cuando es necesario, no se debe dudar en utilizarlos con gentileza para contener el lóbulo cerebeloso ipsilateral al abordaje, pues esta maniobra facilita la visualización del nervio $0^{10,13,15,18-20}$.

La delicada y completa disección del segmento nervioso y de los vasos adyacentes constituye un paso fundamental del procedimiento. Permite la identificación de la compresión vascular o su ausencia ${ }^{10}$. En este punto se decide si se realizará o no neurocirugía asistida por endoscopio. La neuroendoscopia es una herramienta útil que compensa los límites del microscopio quirúrgico ${ }^{21}$. Algunos neurocirujanos han propuesto la utilización de esta herramienta en todos los procedimientos quirúrgicos de $\mathrm{DMV}^{22-24}$. No obstante, basándonos en nuestra experiencia y en la de otros autores ${ }^{9}$, consideramos que la neuroendoscopia debe limitarse a los casos en los que la vía nerviosa es difícil de visualizar, o cuando el área de compresión está situada en un punto de difícil acce$\mathrm{so}^{21}$. Su uso diario, en manos de un cirujano no calificado, podría incluso poner en riesgo la integridad del paciente y aumentar la frecuencia de las complicaciones asociadas a su uso, como hemorragias y lesiones del nervio cerebeloso o del nervio craneal adyacente $e^{19,24,25}$.

\section{Hallazgos quirúrgicos}

La compresión arterial del nervio fue, como en otras series, la principal causa de neuralgia en nuestros pacientes $(61.6 \%)^{4,11,12,18,23,26}$. Según la literatura, la compresión venosa oscila entre el 6 y el $38 \%$ como causa de $\mathrm{NT}^{11,14,23,26}$. En nuestra serie, tres pacientes (12\%) tuvieron compresión venosa, y la colocación de material aislante resolvió satisfactoriamente los episodios de dolor, a diferencia de lo reportado por otros autores, donde la DMV no siempre fue efectiva en los casos de compresión venosa ${ }^{12,27}$.

\section{Resultado terapéutico}

Uno de los aspectos más críticos en la evaluación de cualquier tratamiento, independientemente de su naturaleza, es la posibilidad de evaluar si funciona ${ }^{28}$. Nuestro equipo de trabajo desarrolló una escala para la medición cualitativa de la percepción de la crisis 
neurálgica del paciente desde la intervención quirúrgica hasta el momento de cada evaluación, así como la respuesta del paciente al tratamiento farmacológico, cuando este sea necesario. Esta escala se aplicó cada cuatro meses después de la cirugía durante el primer año de tratamiento, y después cada año. Consideramos que su aplicación es sencilla, breve y accesible, e incluso puede realizarse mediante una entrevista no presencial con el paciente. Considerando el porcentaje de casos sin dolor después de 36 meses (clase A, $69 \%$ ), nuestros resultados están dentro del rango de éxito reportado en la mayoría de las series. Si a estos casos se añade aquellos en los que persistió el dolor pero en los que este cedía con analgésicos no esteroideos $(27 \%)$, nuestra frecuencia de control aceptable del dolor alcanza el $96 \%$, lo que incluso supera a la de otros informes $8,11,15,18-20$. Ninguno de nuestros pacientes requirió reintervención por un control inadecuado del dolor. Las características epidemiológicas y los hallazgos quirúrgicos entre los grupos tratados con éxito y los que no son similares, por lo que es probable que haya otros factores distintos de los estudiados aquí que puedan influir en la erradicación total del dolor. Sin embargo, el número limitado de pacientes dificulta la obtención de conclusiones más precisas.

\section{Complicaciones posquirúrgicas}

Cuando se describen las técnicas quirúrgicas, no todos los autores reportan las complicaciones experimentadas por sus pacientes 9,10 . Consideramos que reportar estos eventos es valioso, ya que permite que otros cirujanos, especialmente los que están en formación, tengan un conocimiento directo de lo que podrían enfrentar en la práctica diaria. Aunque la mayoría de las complicaciones están asociadas con la ejecución de la técnica y por lo tanto con la capacidad del cirujano, la elusión de algunos de estos eventos es en ocasiones inevitable.

Las complicaciones más reportadas después de un procedimiento de DMV son: daño cerebeloso por compresión, fístulas de LCR (debido a un cierre no hermético de la duramadre o a daño en las células mastoideas) y pérdida de la audición debido a una lesión en la rama coclear del VIII nervio craneal ${ }^{13,26}$. La frecuencia de cada una de estas complicaciones varía de una serie a otra. Broggi, et al. (2000) informaron una prevalencia del $6.8 \%$ de hipoacusia en una serie de 250 casos de NT tratados con DMV ${ }^{11}$. La prevalencia en nuestra serie fue ligeramente superior. Sin embargo, en el trabajo de Broggi realizaron monitorización intraoperatoria de los potenciales evocados auditivos, lo que permitía alertar al cirujano cuando el nervio VIII era manipulado o tensado involuntariamente. En nuestro entorno no es plausible disponer de esta tecnología en todos los procedimientos, ya que la mayoría de ellos se realiza en hospitales públicos con recursos limitados. Otros autores reportan tasas de hipoacusia de $1.5-19 \% \%^{12,15,20,29}$.

La incidencia de fístulas de LCR se encuentra dentro de un rango de 2.5 y $15 \%^{11,12,15,18,20}$. Nuestros pacientes se encontraban dentro de este rango con una incidencia del $4 \%$. La parálisis facial se encuentra con una media del $0.6-7.6 \%$ de los $\operatorname{casos}^{18,20,29}$, siendo generalmente una condición temporal. Dos pacientes (8\%) de nuestra serie fueron detectados con déficit del nervio craneal VII, uno de ellos transitorio (House-Brackmann II) y otro con remisión parcial (House-Brackmann III, asociado con infección de herida quirúrgica). No hubo casos de meningitis, infarto cerebral, hematomas, hidrocefalia, parálisis de músculos extraoculares o mortalidad asociada con el procedimiento quirúrgico.

\section{Conclusiones}

La cirugía de DMV trigeminal mediante un abordaje a través del punto asterional es una alternativa con resultados similares al enfoque retrosigmoideo clásico para tratar la NT, pero que añade los beneficios de los principios de la cirugía de mínima invasión. Son necesarios esfuerzos interinstitucionales para realizar estudios prospectivos con mayor número de pacientes y seguimiento a largo plazo, con herramientas de evaluación estandarizadas.

\section{Conflicto de intereses}

Los autores declaran no tener conflictos de interés.

\section{Bibliografía}

1. Patel SK, Liu JK. Overview and history of trigeminal neuralgia. Neurosurg Clin N Am. 2016;27(3):265-76.

2. Eller JL, Raslan AM, Burchiel KJ. Trigeminal neuralgia: definition and classification. Neurosurg Focus. 2005;18(5):E3.

3. van Hecke O, Austin SK, Khan RA, Smith BH, Torrance N. Neuropathic pain in the general population: A systematic review of epidemiological studies: Pain. 2014;155(4):654-62.

4. Chen G, Wang X, Wang L, Zheng J. Arterial compression of nerve is the primary cause of trigeminal neuralgia. Neurol Sci. 2014;35(1):61-6.

5. Hodaie M, Coello AF. Advances in the management of trigeminal neuralgia. J Neurosurg Sci. 2013;57(1):13-21

6. Al-Quliti KW. Update on neuropathic pain treatment for trigeminal neuralgia. The pharmacological and surgical options. Neurosci Riyadh Saudi Arab. 2015;20(2):107-14.

7. Cole CD, Liu JK, Apfelbaum RI. Historical perspectives on the diagnosis and treatment of trigeminal neuralgia. Neurosurg Focus. 2005;18(5):E4. 
8. Jannetta PJ. Arterial compression of the trigeminal nerve at the pons in patients with trigeminal neuralgia. J Neurosurg. 2007;107(1):216-37.

9. Tomasello F, Esposito F, Abbritti RV, Angileri FF, Conti A, Cardali SM et al. Microvascular decompression for trigeminal neuralgia: Technical refinement for complication avoidance. World Neurosurg. 2016:94:26-31.

10. da Silva O, de Almeida C, Iglesio R, de Navarro J, Teixeira M, Duarte K Surgical variation of microvascular decompression for trigeminal neuralgia: A technical note and anatomical study. Surg Neurol Int. 2016;7(22):571.

11. Broggi G, Ferroli P, Franzini A, Servello D, Dones I. Microvascular decompression for trigeminal neuralgia: comments on a series of 250 cases, including 10 patients with multiple sclerosis. J Neurol Neurosurg Psychiatry. 2000;68(1):59-64.

12. Campero A, Campero A, Ajler P. Descompresión microvascular en neuralgia del trigémino: Reporte de 36 casos y revisión de la literatura. Surg Neurol Int. 2014;5(12):441.

13. McLaughlin MR, Jannetta PJ, Clyde BL, Subach BR, Comey $\mathrm{CH}$, Resnick DK. Microvascular decompression of cranial nerves: lessons learned after 4400 operations. J Neurosurg. 1999;90(1):1-8.

14. Kabatas S, Karasu A, Civelek E, Sabanci AP, Hepgul KT, Teng YD Microvascular decompression as a surgical management for trigeminal neuralgia: long-term follow-up and review of the literature. Neurosurg Rev. 2009;32(1):87-93; discussion 93-94.

15. Forbes J, Cooper C, Jermakowicz W, Neimat J, Konrad P. Microvascular decompression: Salient surgical principles and technical nuances. J Vis Exp. 2011;(53):e2590.

16. Day JD, Jordi XK, Manfred T, Takanori F. Surface and superficial surgical anatomy of the posterolateral cranial base: Significance for surgical planning and approach. neurosurgery. 1996;38(6):1079-84.

17. Ucerler H, Govsa F. Asterion as a surgical landmark for lateral cranial base approaches. J Cranio-Maxillofac Surg. 2006;34(7):415-20.

18. Chakravarthi PS, Ghanta R, Kattimani V. Microvascular decompression treatment for trigeminal neuralgia: J Craniofac Surg. 2011;22(3):894-8.
19. Toda H, Goto M, Iwasaki K. Patterns and variations in microvascular decompression for trigeminal neuralgia. Neurol Med Chir (Tokyo). 2015; 55(5):432-41.

20. Venkataramana N, Bansal A, Jagannath $P$, Ravichandra M. Outcome of microvascular decompression for trigeminal neuralgia using autologous muscle graft: A five-year prospective study. Asian J Neurosurg. 2012; $7(3): 125$.

21. Chen MJ, Zhang WJ, Yang C, Wu YQ, Zhang ZY, Wang Y. Endoscopic neurovascular perspective in microvascular decompression of trigeminal neuralgia. J Cranio-Maxillofac Surg. 2008;36(8):456-61.

22. Piazza M, Lee JYK. Endoscopic and microscopic microvascular decompression. Neurosurg Clin N Am. 2016;27(3):305-13.

23. Halpern $\mathrm{CH}$, Lang S-S, Lee JYK. Fully endoscopic microvascular decompression: Our early experience. Minim Invasive Surg. 2013;2013:1-5.

24. Bohman LE, Pierce J, Stephen JH, Sandhu S, Lee JYK. Fully endoscopic microvascular decompression for trigeminal neuralgia: technique review and early outcomes. Neurosurg Focus. 2014;37(4):E18.

25. Marcus HJ, Cundy TP, Hughes-Hallett A, Yang G-Z, Darzi A, Nandi D. Endoscopic and keyhole endoscope-assisted neurosurgical approaches: A qualitative survey on technical challenges and technological solutions. Br J Neurosurg. 2014;28(5):606-10.

26. Teixeira MJ, Siqueira SRDT, Almeida GM. Percutaneous radiofrequency rhizotomy and neurovascular decompression of the trigeminal nerve for the treatment of facial pain. Arq Neuropsiquiatr. 2006;64(4):983-9.

27. Hong W, Zheng X, Wu Z, Li X, Wang X, Li Y, et al. Clinical features and surgical treatment of trigeminal neuralgia caused solely by venous compression. Acta Neurochir (Wien). 2011;153(5):1037-42.

28. Lee JYK. Measurement of trigeminal neuralgia pain. Neurosurg Clin N Am. 2016;27(3):327-36.

29. Oesman C, Mooij JJA. Long-term follow-up of microvascular decompression for trigeminal neuralgia. Skull Base. 2011;21(5):313-22. 